\title{
Bioinformation
}

\section{Model based design of inhibitors for c-jun}

\author{
Pallavi Chauhan $^{1, *}$, Madhvi Shakya ${ }^{2}$ \\ ${ }^{1}$ Department of Bioinformatics, ${ }^{2}$ Department of Mathematics, MANIT, Bhopal, MP, India; Pallavi Chauhan - Email: \\ pallavi.chauhan2006@gmail.com; Phone: +91-9893783627; Fax: 0755- 2670562; *Corresponding author
}

Abstract:

Received October 01, 2009; Revised November 07, 2009; Accepted November 18, 2009; Published November 26, 2009

Literature shows that various molecular cascades are activated by stress, UV rays and pollutants leading to wrinkle formation of the skin. These cascades start from five types of receptors (EGFR, PDGFR, PAFR, IL1R, TNFRB) and terminate with the production of matrix metalloproteinase's, which degrades collagen leading to wrinkle formation. Signaling pathway leading to wrinkle formation showed that c-jun is involved in these cascades. Therefore, c-jun is the preferential choice for inhibition to reduce the intensity of collagen degradation. Hence, the 3D structure of c-jun was modeled using segment based homology modeling by MODELLER 9v5. Evaluation of the constructed model was done by PROCHECK, WHAT CHECK and through RMSD/RMSF calculations. Ligands for the inhibitory sites were designed using LIGANDSCOUT. The interaction study of ligand and receptor was performed by AUTODOCK. A library of analogues was constructed for three known inhibitory sites. The receptor-analogue study was performed using the software MOLEGRO Virtual Docker. The analogues constructed from the designed novel reference ligands showed good binding with the receptor binding sites. It should be noted that these predicted data should be validated using suitable assays for further consideration.

Keywords: wrinkle formation; homology modeling; docking; inhibitors for c-jun

\section{Background:}

Skin ageing is a hot research topic. Skin research has led to the development many products to keep skin younger and healthier for a long period. There are many preparations like Botox injections, laser treatment, fat graft implantation, etc., for skin ageing. However, these products are often expensive and usually have side effects. Hence, there is a need to analyze the molecular pathways for skin ageing and to inhibit potential targets. There are various symptoms for skin ageing but one of the most prominent sign of skin ageing is wrinkle formation. Wrinkle appears on the skin both due to intrinsic and chronological skin ageing (occurs with the passage of time) and premature skin ageing (influence of external factors like UV rays, stress and pollution). Data suggest that there are five receptors (EGFR, PDGFR, PAFR, IL1R and TNFRB) that are activated by stress. They stimulate their corresponding adapter proteins, mitogen activated protein kinases (MAPKs) and transfer the signal down to the nucleus to transcription factor activation protein 1 (AP-1). AP-1 further induces the transcription of matrix metalloproteinase's (MMPs).

$\operatorname{MMPs}(1,3,9)$ degrade collagen. The excessive degradation of collagen leads to loss of skin integrity towards wrinkle formation. Signaling cascade studies show that EGFR, PDGFR and PAFR through MAPK1 bring activation of AP-1. TNFRB through MAPK9 and IL1R through MAPK8 activation of AP-1 are known. However, different MAPKs were involved leading to activation of AP-1. Hence, if collagen degradation, activated by different stressors through different receptors is to be inhibited; AP-1 should be targeted [1]. AP-1 is made up of two subunits, namely c-fos and c-jun. It is known that c-jun expression is limiting AP-1 activation in human skin as c-fos concentration is constant, while c-jun concentration increases in photo-aged skin and old skin [2]. Moreover, c-fos-regulated genes exert a protective function and its deficiency makes the cells hypersensitive to a broad spectrum of DNA-damaging agents. Therefore, c-jun is a preferential choice for inhibition. c-jun is 331 residues long. It consists of a leucine zipper domain and a basic region. c-jun interacts with DNA by the leucine zipper domain.

In c-jun, the transcription activation domain residues are 91-186, the transcription repression domain residues are 31-57 and the promoter region for $\mathrm{T}$ antigen-dependent DNA unwinding (required for the initiation of polyoma virus DNA replication) residues are 91-186. The region 241- 252 residues of c-jun can increase DNA bending and binding at the AP-1 site [3]. Residues 252-281 in c-jun are the basic sub-domain, which is responsible for the sequence specific recognition site of DNA [4]. Studies performed so far have targeted MAPKs to inhibit the activation of c-jun. It has been shown that three types of
MAPK are involved in collagen degradation. Therefore, to completely inhibit the degradation of collagen (wrinkle formation) activated by various stressors, c-jun is targeted. However, the complete structure for c-jun is still not available for inhibitor design. Therefore, homology modeling is performed to construct the 3D structure for cjun followed by inhibitor design through docking.

\section{Methodology:}

\section{Homology modeling:}

3D structure modeling is the first step towards successful in silico drug designing. A suitable template with sufficient query sequence length coverage and sequence identity is not as yet available. Therefore, segment based homology modeling approach is used in this study. Fold recognition was employed for loop modeling in this procedure. BLAST and PSI BLAST were used for the selection of PDB templates and templates with $>40 \%$ sequence identity with the target sequence were selected (Table 1 in supplementary material). LOMETS (Local Meta Threading Server) was used to select templates for loop modeling. The server selects fold not only on the basis of secondary structure but also considers mutations, solvent accessibility and pairwise residue contacts [5]. Subsequently, the target sequence and templates were aligned using MODELLER 9v5 and thus, a 3D model was constructed. The constructed model was energy minimized in GROMACS force field using Steepest Descent Minimization Algorithms. The model was evaluated using PROCHECK, WHAT CHECK and RMSD, RMSF calculations. RMSD and RMSF calculations are good indicators of the uncertainty in the atomic coordinates and the favorable value is within $0.2 \mathrm{~nm}$. PROCHECK validates the model for covalent bond distances, angles, atom nomenclature and stereo-chemistry [6]. What-check was used to evaluate the folding pattern of the model [7].

\section{Ligand and receptor interaction:}

Inhibitors are not yet available for c-jun. Therefore, it is important to design suitable inhibitors for c-jun. Ligands were designed using LIGAND SCOUT 2.02. The designed ligand and receptor interaction at desired position are performed using AUTODOCK version 1.5.2. Ligand analogues have been constructed using CHEMSKETCH and docking was performed by Molegro Virtual Docker (MVD). We used MVD because it showed higher docking accuracy than other state-ofthe-art docking products (MVD: $87 \%$, Glide: $82 \%$, Surflex: $75 \%$, FlexX: 58\%) in the market [8]. During virtual screening, the following parameters were fixed: number of runs 10 , population size 50 , crossover rate 0.9 , scaling factor 0.5 , maximum iteration 2,000 and grid resolution 0.30 . The docked results were evaluated on the basis of binding affinity, moledock score and re-ranking. Binding affinity 
$(\mathrm{kg} / \mathrm{mol})$ is measured on the basis of known experimental data. The MolDock scoring function takes into consideration directional hydrogen bonding and charges. The docking scoring function, E score, is defined by the energy terms in equation 1 (see supplementary material). Re-rank-score in MVD provides an estimate for interaction.

\section{Discussion:}

\section{Model evaluation:}

The constructed 3D model is shown in Figure 1. The results of PROCHECK parameters like Ramachandran plot (90.8 core, 7.7 allowed, 1.1 generous, 0.4 disallowed), main chain (6), side chain (5), bond length (97.8), bond angle (93.4) and list of all planar groups (100) were within favorable limits for the constructed model. The folding patterns evaluated by WHAT CHECK is similar to the comparative study of various tools for secondary structure prediction (GOR, JFRED, HNN, NN predict, PORTER, SOPMA and SSpro). Therefore, the constructed model holds good for folding patterns. The RMSD value calculated for model backbone after first fit to backbone at 9000 cycles (9ps time) gave a constant deviation of $0.125 \mathrm{~nm}$. RMSF value calculated for the model was between $0.025-0.125 \mathrm{~nm}$. The RMSD and RMSF values calculated were within the favorable limit of $0.2 \mathrm{~nm}$.

\section{Inhibition site:}

Literature studies have shown that phosporylation and consequently dephosphorylation brings about the activation of c-jun. The $\mathrm{NH}_{2}$ terminal phosphorylation of c-jun in response to growth factors and $\mathrm{UV}$ rays is responsible for $\mathrm{COOH}$ terminal dephosphorylation. The $\mathrm{NH}_{2}$ terminal amino acid that undergoes phosphorylation is serine 63 , serine 73 and threonine 91. This initiates dephosphorylation of residues threonine 231, serine 243 and serine 249. Dephosphorylation decreases the electrostatic repulsions between the phosphate groups on c-jun and the DNA-binding site, hence enhancing c-jun binding on DNA [9-11]. Hence, the 3 phosphorylation sites should be inhibited simultaneously in order to suppress the binding of c-jun on DNA and transcription of MMPs.

\section{Ligand Design:}

Novel ligands have been designed with ligand scout 2.02. The constructed ligand that showed interaction at desired phosphorylation inhibition sites by AUTODOCK were considered as reference ligand. We developed 3 reference ligands for the three inhibitory sites. Figure 2 shows the interactions of the reference ligand at the desired site on receptor. The reference ligand for site 1 shows interaction with ser 63 (desired) and asp 65. Similarly, reference ligand designed for site 2 shows interaction with ser 73 (desired) and glu77. The reference ligand for site 3 shows interaction with ser 83 , gly 86, his 87 , thr 90 and thr 91 (desired). The $\mathrm{R}_{1}$ symbol on each reference ligand identifies the site, where desired interaction is seen between ligand and receptor. All the three reference ligands interacted well at the desired sites for corresponding phosphorylation.

\section{Virtual screening:}

The reference ligands however showed desired interaction on receptor but have low binding affinity with the receptor as demonstrated by MVD. Various analogues have been constructed by adding different functional groups (from + inductive effect to -inductive effect) at the sites $R_{1}$ and $R_{2}$ to obtain ligands having greater binding affinity with the receptor. Additional functional groups at $\mathrm{R}_{1}$ increased the affinity of ligand at the desired inhibitory site. However, attachment of different functional groups at $\mathrm{R}_{2}$ created an environment by interacting with neighboring residues as shown in Figure 3. The designed analogues also gave better calculations for binding affinity, moldock score and re-ranking score than their corresponding reference ligands, listed in Table 2 (See supplementary material). Thus, the designed analogues showed interaction at desired inhibitory site with good docking score, for potential testing as a ligand.

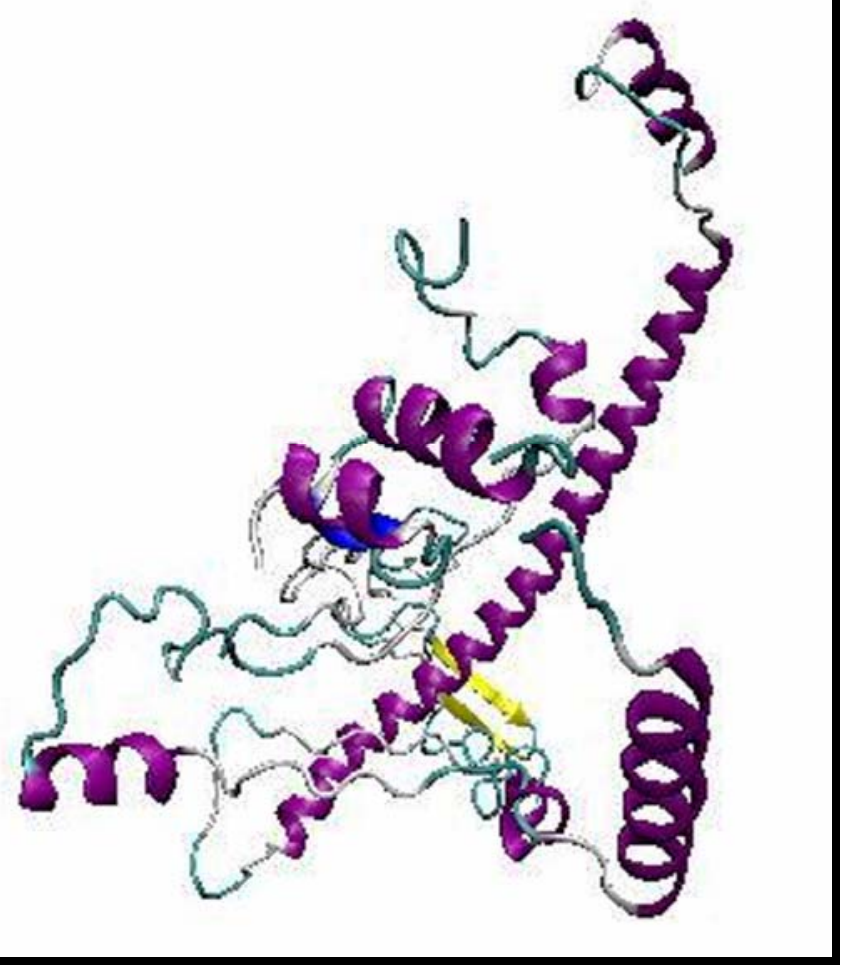

Figure 1: 3D model of c-jun. The model is generated using MODELLER. 


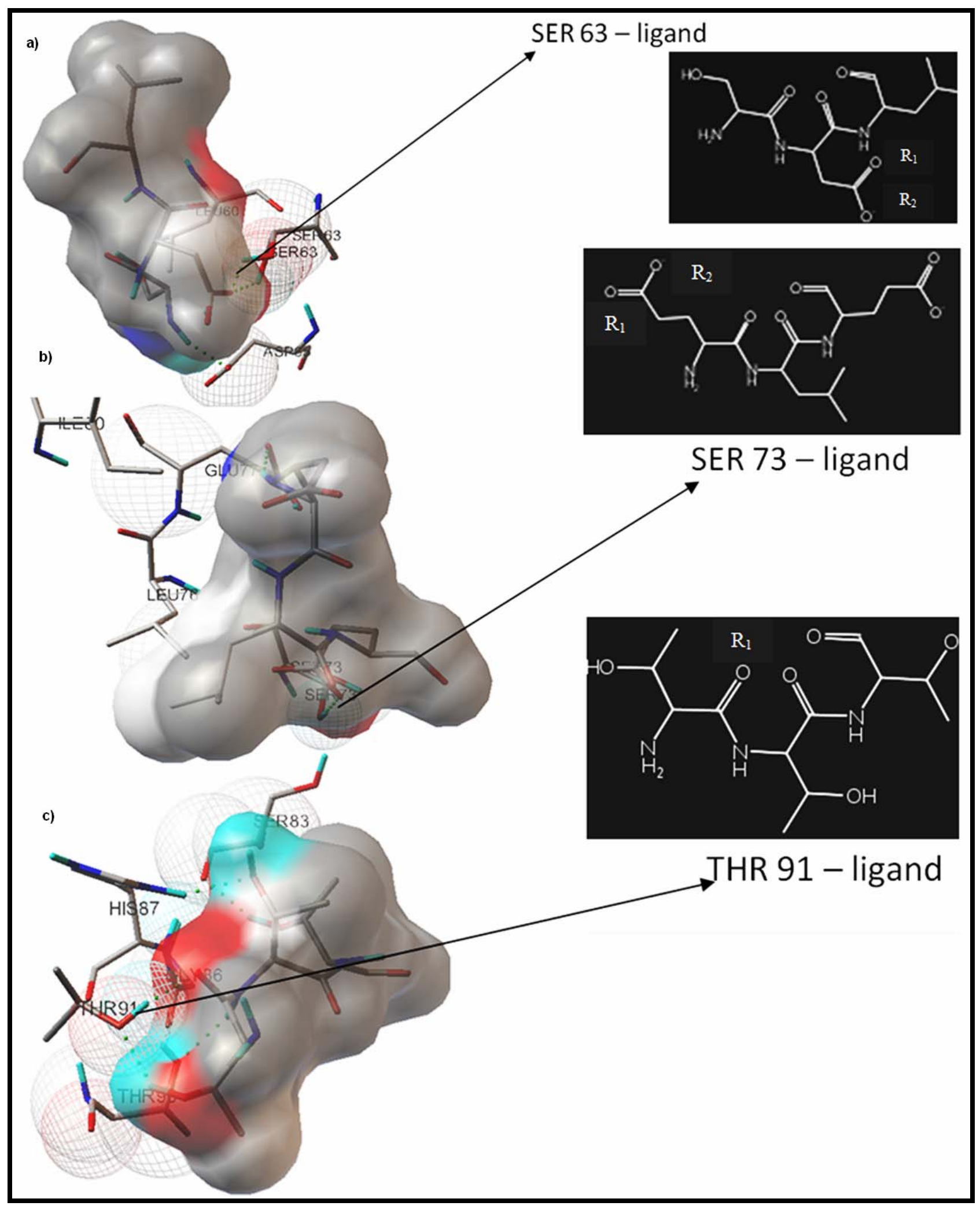

Figure 2: The docking view showing the binding of designed reference ligands with receptor at desired site. 


\section{Bioinformation}
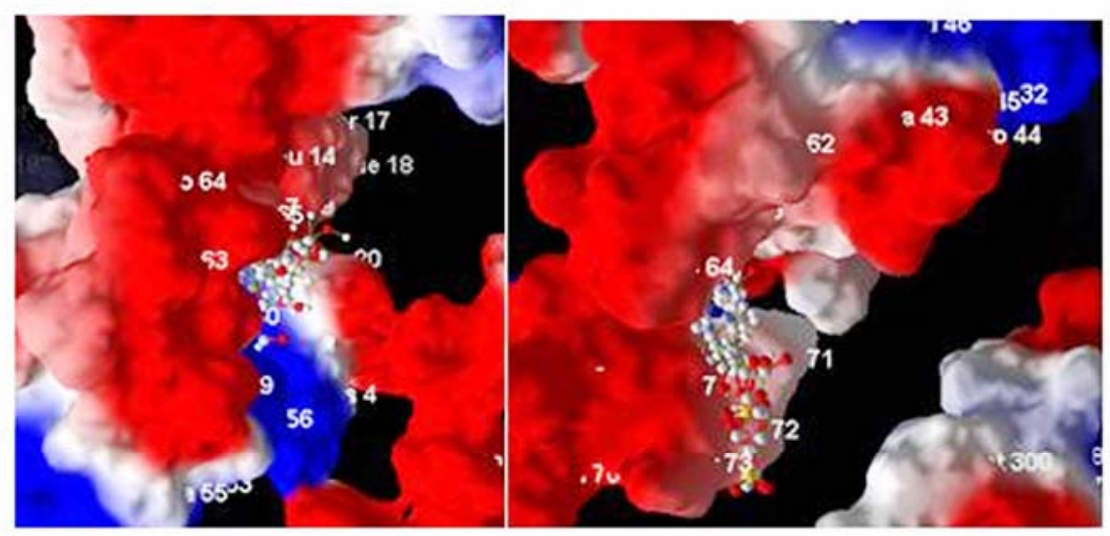

Site I (Ser 63)

Site II (Ser 73)

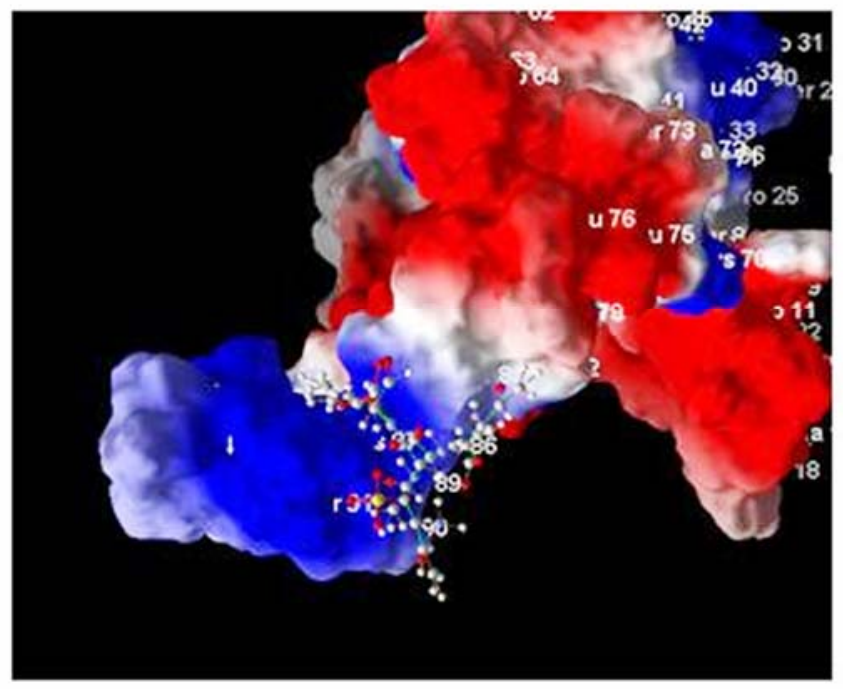

Site III (Thr 91)

Figure 3: The docking view of analogues and receptor for three different inhibition site.

\section{Conclusion:}

Wrinkle formation is one of the most prominent signs of skin ageing. There is a tremendous demand for products that can completely inhibit collagen degradation. However, these products have side effects. Therefore, it is important to design inhibitors for targets like c-jun that are associated with wrinkle formation. Here, we show the use of modeling and virtual screening in the potential design of inhibitors for c-jun. It should be noted that these predicted data should be validated using suitable assays for further consideration in future studies.

\section{References:}

[1] P Chauhan et al., IJDVL.75: 463 (2009) [PMID: 19736424]

[2] GJ Fisher et al., Arch Dermatol.138: 1462 (2002) [PMID 12437452]
[3] K Tom et al., EMBO J. 16: 2907 (1997) [PMID: 9184234]

[4] D.Krebs et al., Eur J Biochem. 231: 370 (1995) [PMID: 7635148]

[5] S Wu et al., Nucleic Acids Res. 35: 3375 (2007) [PMID: 17478507]

[6] RA Laskowski et al., J. Biomolecular NMR 8: 477 (1996) [PMID: 9008363]

[7] RWW Hooft et al., Nature 381: 272 (1996) [PMID: 8692262]

[8] R Thomsen et al., J Med Chem., 49: 3315 (2006) [PMID: 16722650]

[9] G Athanasios et al., The EMBO J. 14: 2014 (1995) [PMID: 7744008]

[10] T Smeal et al., Mol Cell Biol. 12: 3507 (1992) [PMID: 1630458]

[11] B Derijard et al., Cell 76: 1025 (1994) [PMID: 8137421]

Edited by $P$. Kangueane

Citation: Chauhan \& Shakya, Bioinformation 4 (6): 223-228 (2009) License statement: This is an open-access article, which permits unrestricted use, distribution, and reproduction in any medium, for noncommercial purposes, provided the original author and source are credited. 


\section{Supplementary material:}

$$
\begin{aligned}
& \mathbf{E}_{\text {Score }}=\mathbf{E}_{\text {inter }}+\mathbf{E}_{\text {intra }} \\
& E_{\text {inter }}=\sum_{i \in \text { iggand }} \sum_{j \in \text { protein }}\left[E_{P L P}\left(r_{i j}\right)+332.0 \frac{q_{i} q_{j}}{4 r_{i j}{ }^{2}}\right]
\end{aligned}
$$

\section{Equation 1}

Where $E_{P L P}$ is a piecewise linear potential and the second term describes the electrostatic interactions between charged atoms

$$
E_{\text {intra }}=\sum_{i \in \text { ligand }} \sum_{j \in \text { ligand }} E_{P L P}\left(r_{i j}\right)+\sum_{\text {flexible bonds }} A\left[1-\cos \left(m \cdot \theta-\theta_{0}\right)\right]+E_{\text {clash }}
$$

Where double summation is between all atom pairs in the ligand, the second term is a torsional energy, $\theta$ is the torsional angle of the bond and $E_{\text {clash, }}$, assigns a penalty of 1000 if the distance between two heavy atoms is less than $2.0 \mathrm{~A}$.

Table 1: List of PDB templates used for segment based homology modeling

\begin{tabular}{cccc}
\hline Tools used/purpose & $\begin{array}{c}\text { Selected } \\
\text { templates } \\
\text { (PDB ID) }\end{array}$ & $\begin{array}{c}\text { Length covered by PDB template } \\
\text { on the protein sequence }\end{array}$ & $\begin{array}{c}\text { Sequence/ fold identity between the PDB } \\
\text { and reference protein sequence }\end{array}$ \\
\hline BLAST \& PSI BLAST & 2AMI & $1-5$ & $100 \%$ \\
(Selection of PDB templates for & 2PZJ & $6-17$ & $66 \%$ \\
model construction) & $1 \mathrm{FCY}$ & $72-96$ & $46 \%$ \\
& $1 \mathrm{AF} 2$ & $173-204$ & $40 \%$ \\
& $1 \mathrm{NNM}$ & $254-310$ & $58 \%$ \\
LOMETS & 1U7O & $314-331$ & $50 \%$ \\
(To fill the loops, regions not & 1ERD & $30-69$ & $>50 \%$ \\
covered by BLAST PDB ) & 1DEV & $97-170$ & $206-246$ \\
\hline
\end{tabular}

Table 2: Comparative study of binding affinity, moldock score and re-ranking score of reference ligand with analogues for three inhibitory sites

\begin{tabular}{ccccc}
\hline \multicolumn{5}{c}{ SITE I (ser 63) } \\
\hline R1 & R2 & Binding affinity & Moldock score & Reranking score \\
\hline O & O & $-\mathbf{- 1 2 . 2 8 0 5}$ & $\mathbf{- 8 7 . 5 8 3 4}$ & $-\mathbf{7 2 . 9 5 4 4}$ \\
COCl & O & -16.5375 & -111.701 & -84.5607 \\
COCH3 & O & -14.6547 & -117.062 & -83.3443 \\
OSO3H & O & -13.0595 & -120.418 & -85.0854 \\
COOEt & O & -16.4993 & -120.514 & -92.2664 \\
66H5O & O & -15.206 & -122.575 & -90.6628 \\
H2OPO2 & O & -13.9347 & -130.74 & -98.4258 \\
C6H5OAc & O & -16.7329 & -134.646 & -99.2073 \\
C6H5NO & O & -14.3909 & -130.665 & -95.9775 \\
C6H5CHO & O & -11.7646 & -134.708 & -90.5285 \\
C6H5OAc & CHO & -20.9032 & -120.961 & -93.2101 \\
C6H5OAc & COCH3 & -19.8917 & -143.012 & -103.415 \\
C6H5OAc & ONO & -24.7791 & -142.664 & -103.735 \\
C6H5OAc & OCHO & -19.8626 & -146.693 & -98.6499 \\
C6H5OAc & SO2H & -19.0394 & -144.512 & -103.86 \\
C6H5CHO & COOCH3 & -19.4519 & -124.963 & -96.1255 \\
C6H5CHO & CONH2 & -17.2812 & -144.175 & -110.27 \\
C6H5CHO & COCl & -19.116 & -120.203 & -89.3363 \\
C6H5CHO & NO & -17.9934 & -135.044 & -103.948 \\
C6H5CHO & OAc & -19.905 & -138.993 & -105.387 \\
COPh & CONH2 & -15.1967 & -138.589 & -101.398 \\
COPh & COCl & -18.0481 & -145.42 & -101.962 \\
COPh & NHAc & -14.2186 & -147.456 & -107.879 \\
H2PO3 & ONO & -15.8022 & -121.942 & -96.432 \\
H2PO3 & NHAc & -14.8584 & -125.068 & -97.9593 \\
H2PO3 & OAc & -20.6259 & -128.349 & -102.712 \\
H2PO3 & COOCH3 & -20.9547 & -129.354 & -101.996 \\
\hline & & & & \\
\hline & & & & \\
\hline
\end{tabular}




\section{Bioinformation}

\begin{tabular}{|c|c|c|c|c|c|}
\hline \multicolumn{6}{|c|}{ SITE II (ser 73) } \\
\hline R1 & R2 & \multicolumn{2}{|c|}{ Binding affinity } & Moldock score & Reranking score \\
\hline $\mathrm{O}$ & $\mathrm{O}$ & \multirow{2}{*}{\multicolumn{2}{|c|}{$\begin{array}{c}\mathbf{- 1 0 . 0 9 7 5} \\
-12.601\end{array}$}} & -97.968 & -75.8075 \\
\hline $\mathrm{H} 2 \mathrm{PO} 3$ & $\mathrm{O}$ & & & -100.64 & -87.7476 \\
\hline NO & $\mathrm{O}$ & \multicolumn{2}{|c|}{-12.4778} & -110.199 & -91.3151 \\
\hline $\mathrm{COOPh}$ & $\mathrm{O}$ & \multicolumn{2}{|c|}{-19.5081} & -114.199 & -96.0297 \\
\hline OCH3 & $\mathrm{O}$ & \multicolumn{2}{|c|}{-15.5548} & -115.03 & -93.0747 \\
\hline $\mathrm{OAc}$ & $\mathrm{O}$ & \multicolumn{2}{|c|}{-16.2034} & -118.937 & -94.9341 \\
\hline $\mathrm{C} 6 \mathrm{H} 5 \mathrm{COOCH} 3$ & $\mathrm{O}$ & \multicolumn{2}{|c|}{-20.6526} & -119.826 & -94.7233 \\
\hline С6H5OPO3H2 & $\mathrm{O}$ & \multicolumn{2}{|c|}{-20.0411} & -105.614 & -89.0779 \\
\hline C6H5SO2H & $\mathrm{O}$ & \multicolumn{2}{|c|}{-17.75} & -119.0791 & -95.3803 \\
\hline C6H5OCHO & $\mathrm{O}$ & \multicolumn{2}{|c|}{-20.1399} & -124.793 & -91.277 \\
\hline $\mathrm{C} 6 \mathrm{H} 5 \mathrm{CHO}$ & $\mathrm{O}$ & \multicolumn{2}{|c|}{-18.8208} & -129.938 & -102.681 \\
\hline COOEt & $\mathrm{CHO}$ & \multicolumn{2}{|c|}{-14.8759} & -112.365 & -93.4722 \\
\hline COOEt & $\mathrm{COCl}$ & \multicolumn{2}{|c|}{-19.3856} & -109.854 & -94.6401 \\
\hline $\mathrm{C} 6 \mathrm{H} 5 \mathrm{CHO}$ & $\mathrm{COOCH} 3$ & \multicolumn{2}{|c|}{-24.4418} & -116.134 & -90.7422 \\
\hline $\mathrm{C} 6 \mathrm{H} 5 \mathrm{CHO}$ & $\mathrm{COCH} 3$ & \multicolumn{2}{|c|}{-25.0494} & -110.399 & -77.1874 \\
\hline $\mathrm{C} 6 \mathrm{H} 5 \mathrm{CHO}$ & COOEt & \multicolumn{2}{|c|}{-24.4518} & -109.6 & -83.6871 \\
\hline $\mathrm{C} 6 \mathrm{H} 5 \mathrm{CHO}$ & $\mathrm{CONH} 2$ & \multicolumn{2}{|c|}{-22.3118} & -107.413 & -74.9499 \\
\hline C6H5OCHO & $\mathrm{CHO}$ & \multicolumn{2}{|c|}{-21.9882} & -121.115 & -93.1812 \\
\hline C6H5OCHO & $\mathrm{COOCH} 3$ & \multicolumn{2}{|c|}{-27.3662} & -116.949 & -89.2061 \\
\hline C6H5OCHO & $\mathrm{COCH} 3$ & -29. & 138 & -110.29 & -75.225 \\
\hline \multicolumn{6}{|c|}{ SITE III (Thr 91) } \\
\hline R1 & \multicolumn{2}{|c|}{ Binding affinity } & \multicolumn{2}{|c|}{ Moldock score } & ranking score \\
\hline $\mathrm{O}$ & \multirow{2}{*}{\multicolumn{2}{|c|}{$\begin{array}{l}-\mathbf{1 0 . 6 5 2 5} \\
-138865\end{array}$}} & \multicolumn{2}{|c|}{-62.9956} & -42.2497 \\
\hline $\mathrm{COOCH} 3$ & & & & 221 & -70.5025 \\
\hline $\mathrm{COPh}$ & \multicolumn{2}{|c|}{-12.9626} & \multicolumn{2}{|c|}{-86.2756} & -66.2633 \\
\hline ONO & & 588 & -93 & 583 & -70.182 \\
\hline OAc & & 479 & -93 & 005 & -70.1516 \\
\hline $\mathrm{SO} 2 \mathrm{H}$ & & 113 & & 338 & -68.6467 \\
\hline NHAc & & 655 & -94 & 558 & -74.6504 \\
\hline $\mathrm{OSO} 3 \mathrm{H}$ & & 516 & -96 & 543 & -71.6728 \\
\hline OPO3H2 & & 183 & & & -68.2773 \\
\hline $\mathrm{C} 6 \mathrm{H} 5 \mathrm{CHO}$ & & 956 & -98 & 812 & -74.4093 \\
\hline C6H5NO & & 098 & -10 & 005 & -73.7687 \\
\hline C6H5COCH3 & & 466 & & 861 & -75.4764 \\
\hline C6H5OAc & & 636 & & 187 & -83.7989 \\
\hline $\mathrm{C} 6 \mathrm{H} 5 \mathrm{COOCH} 3$ & & 597 & & & -78.0367 \\
\hline $\mathrm{COOPh}$ & & 241 & -11 & 306 & -83.0351 \\
\hline
\end{tabular}

